\title{
Steps in Predicting the Relationship of Yield on Fungicide Dose
}

\author{
N. D. Paveley, R. Sylvester-Bradley, R. K. Scott, J. Craigon, and W. Day
}

First author: ADAS High Mowthorpe, Duggleby, Malton, North Yorkshire YO17 8BP; second author: ADAS Boxworth, Boxworth, Cambridge CB3 8NN, U.K.; third and fourth authors: University of Nottingham, Sutton Bonington Campus, Loughborough, Leicestershire LE12 5RD, U.K.; and fifth author: Silsoe Research Institute, Wrest Park, Silsoe, Bedford MK45 4HS, U.K.

R. K. Scott is deceased.

Accepted for publication 9 April 2001.

\begin{abstract}
Paveley, N. D., Sylvester-Bradley, R., Scott, R. K., Craigon, J., and Day, W. 2001. Steps in predicting the relationship of yield on fungicide dose. Phytopathology 91:708-716.

A set of hypothetical steps has been defined, which links fungicide dose to marketable yield, whereby (i) increasing dose decreases symptom area, according to a dose-response curve, (ii) decreased symptom area increases crop green area index (GAI), (iii) increasing GAI increases fractional interception of photosynthetically active radiation, (iv) increased fractional interception increases crop dry matter accumulation, and (v)

entiating with respect to the ratio of fungicide cost over yield value, an analytical solution was obtained for the economic optimum dose. Taking published ranges of parameter values for the Septoria tritici wheat pathosystem as an example, yield-response curves and optimum doses were biologically plausible when compared with data from four field experiments. The analytical and empirical results imply that the dose required to optimize economic return will vary substantially between sites, seasons, and cultivars. Sensitivity analyses identified parameters describing specific facets of disease severity, fungicide efficacy, and assimilate partitioning as most influential in determining the dose optimum.
\end{abstract} yield increases, depending on the partitioning of dry matter to the marketable fraction. One equation represented all five steps. By integrating this equation for light interception during the yield forming period and differ-

Waggoner and Berger (52) described how foliar disease severity might relate to crop growth and yield through effects on light capture. Their application of the Beer's law analogy $(29,31)$ to quantify the effect of disease-induced loss of green canopy area on crop growth has proved helpful for analysis of disease-yield loss relationships in a number of pathosystems $(14,27,28,32)$.

Recently, Paveley et al. (34) quantified how fungicides reduce foliar disease severity in wheat by delaying and reducing epidemic onset and rate, the magnitude of the effect being dependent on both spray timing and dose. Increasing dose increments caused decreasing increments of effect, resulting in a response curve (8) of disease severity on dose. Optimum spray timings, giving the minimum dose for a given reduction in severity, related closely to the pattern of development of the wheat canopy and are, therefore, potentially predictable from observations of crop development. However, the dose required to optimize economic return has not been adequately explored; there are likely to be important effects arising from variation in the relationship between dose and disease, as well as between disease and yield loss. In this respect, the literature lags behind the sophistication of practical decision making. Growers of temperate arable crops are already deciding to apply less than the dose recommended by the manufacturers $(40,46)$, although there is little theory to guide the adjustment of dose according to the state of a particular crop.

Models coupling disease and yield have been advocated $(3,37)$ and developed $(21,23,51)$ by several workers. In this study, a method is proposed to relate dose to yield by coupling the principles of light capture in crop canopies with descriptions of disease on dose. We developed a simple hypothetical framework, through which the effects of variation in fungicide, pathogen, host, and en-

Corresponding author: N. D. Paveley; E-mail address: neil.paveley@ adas.co.uk

Publication no. P-2001-0522-01R

(C) 2001 The American Phytopathological Society
Additional keywords: coupled models, economic threshold, resource capture.

vironment might be assessed, in order to identify the prime influences on the optimum fungicide dose, and hence to guide experimentation and measurement.

\section{THEORY AND APPROACHES}

The description below of the processes linking yield and fungicide dose is based on the interaction of foliar diseases with the leaf canopy of wheat, and is intended to be sufficiently simple to allow an analytical definition of the economically optimum dose. Some of the assumptions required for this approach do not necessarily apply to other pathosystems.

Relationship of grain yield on total dry matter accumulation. Grain constitutes approximately half of the final dry weight of a wheat crop and arises largely from the second half of the dry matter to be formed. The life cycle of wheat can therefore be divided into a "foundation period," during which the photosynthetic and grain-bearing structures are formed, followed by a "yield forming period," during which materials destined to be grain are assimilated. This approximation is supported by earlier work, which suggested that analysis of disease and yield loss should focus on the period when the harvested portion of a crop is growing rapidly $(5,6,10,19)$.

The relationship of grain dry matter on total dry matter (DM) can be represented by a straight line with a slope $\left(\phi_{g}\right)$, which represents the fraction of current above ground dry matter that will ultimately form grain. In approximate terms, the slope will increase from zero to one during the transition between the foundation and yield forming periods. For the purposes of this paper, in which the interest is solely in accumulation of dry matter during the yield forming period (defined here as the period from anthesis to maturity), partitioning might be considered constant and the intercept zero, therefore, grain DM $=\phi_{g} \times$ total crop DM. Both axes of the relationship have units of weight per unit of ground area occupied by the crop. 
Relationship of total dry matter on light interception. Crops accumulate dry matter in proportion to the amount of photosynthetically active radiation (PAR) that their green tissues intercept (12). Waggoner and Berger's analysis (52) suggested that radiation use efficiency (RUE) was conserved in the remaining green leaf area of diseased crops, a finding subsequently confirmed in other pathosystems $(43,50)$. However, Johnson (22) noted that disease-induced effects on RUE were possible, and he simplified Boote et al.'s (3) categories of pest effects on crop carbon fixation into type I and type II pathosystems, depending on whether the effect on yield was predominantly through RUE or radiation interception, respectively. Effects on the efficiency of the remaining green area have been reported $(2,13,36)$, but there remains no clear evidence of such effects predominating, or that any fungal foliar pathosystem should be categorized clearly as type I. Therefore, the question of the relative importance of effects on RUE and radiation interception remains.

Here, the relationship of total dry matter on light energy intercepted is represented as a straight line with an intercept at the origin and a slope $\left(\varepsilon_{p}\right)$ equivalent to the RUE in grams per megajoule of PAR. Therefore, during any period when efficiency might be considered constant, total crop DM $=\varepsilon_{p} \times$ PAR intercepted.

Relationship of light interception on green area. PAR intercepted is conventionally related to the size of the intercepting surface, the green area index (GAI), through Beer's law (31): $f=1-e^{-k_{1} L}$, where $f=$ fraction of PAR intercepted, $k_{1}$ is the dimensionless extinction coefficient for PAR, and $L=$ GAI (dimensionless).

This assumes that necrotic leaf area does not intercept radiation, which might otherwise be intercepted usefully by green tissue lower down the canopy. The constant $k_{1}$ is relatively stable for modern wheat crops (48). The fraction $f$ is applied to incident PAR $\left(I_{p}\right)$ (megajoule per square meter per day) to provide the amount of PAR intercepted. Because incident radiation is expressed as a rate, the relationships described thus far need to be quantified as light intercepted and dry matter increase during some specified period. During any period when $L$ and $k_{1}$ might be considered as constant, intercepted PAR $=I_{p}\left(1-e^{-k_{1} L}\right)$.

Relationship of green area on symptom area. The development of a simple field technique to measure total leaf area (5) allows the proportion of leaf area affected by symptoms to be multiplied by total leaf area (green and dead) and expressed as an area index (symptom area index [SAI]). This approach requires that all of the impact of symptoms on green area is accounted for (44), but the area lost to defoliation (52) is not present to be assessed so underestimation is likely to result. To account for this, the relationship of GAI on SAI can be represented by a straight line with a negative slope and an intercept at the GAI of the healthy control $\left(L_{h}\right)$. The slope $(D)$ would be steeper than unity where there was defoliation associated with disease-induced necrosis. At any time $(t)$, or during any period when healthy green area could be considered constant and when unattributable loss of green area remains a constant proportion of symptom area, then $L=L_{h}-D \times \mathrm{SAI}$.

Although underestimation of GAI loss is accounted for, interception of light by symptom area is not. We neglected the latter because badly diseased leaves and necrotic lesions within leaves are lost over periods that are substantially shorter than the leaf duration, and many diseases affect mainly older leaves, usually at the bottom of the canopy (28). More rigorous forms of the Beer's law function may be required when these are not the characteristics of the pathosystem under consideration (28).

Relationship of symptom area on fungicide dose. Fungicide efficacy has long been quantified with dose-response curves (8, 20). A fungicide is applied at a range of doses, and the effect on the pathogen or symptom expression is quantified. An exponential function provides a parsimonious description of the relationship between disease severity on dose for foliar diseases of wheat (34). Crucially, the relationship of symptoms to green area, described in the previous step, dictates that the $y$ axis of the present step must be expressed as SAI if the aim is to relate dose and symptoms to yield. Thus, at any time $(t)$, or for any period during which symptom area might be considered constant, the relationship of symptom area on dose could be represented as $S=S_{a}+S_{b} e^{-k_{2} \cdot d o s e}$, where $S=$ SAI, $S_{a}=$ SAI with an infinite fungicide dose (the lower asymptote), $S_{b}=$ the amount of SAI that might potentially be controlled with an infinite dose (hence, $S_{a}+S_{b}=$ untreated SAI), and $k_{2}$ defines the rate of change in $S$ with increasing dose (square meter per dose). Dose can be described in absolute terms (gram of active ingredient per square meter) or as a proportion of the recommended dose set by the manufacturer (11), with the value for $k_{2}$ varying accordingly.

Steps from fungicide dose to yield. Each of the relationships previously described can be arranged so the dependent variable from one relationship becomes the independent variable for the next, and fungicide input relates to yield output through linked steps (Fig. 1), whereby (i) increased dose decreases symptom area by diminishing increments, (ii) decreased symptom area translates to increased GAI, (iii) increased GAI increases the interception of PAR, (iv) increased interception of PAR increases crop dry matter, according to RUE, and (v) increased total dry matter increases yield, according to the partitioning of assimilate to grain.

In algebraic form, the dependent variable, yield (gram per square meter), can be described in terms of the independent variable, dose (gram per square meter), through one equation:

$$
\text { Grain DM }=\phi_{g} \varepsilon_{p} I_{p}\left(1-e^{-k_{1}\left[L_{h}+\left[D\left(S_{a}+S_{b} e^{-k_{2} \text { dose }}\right)\right]\right\}}\right)
$$

Steps 1 and 3 are both exponential and, because symptoms subtract from green area, both relationships act in concert to cause curvature in the yield on dose relationship. Because the economic cost of fungicide increases in proportion to dose, and economic output increases in proportion to yield, it follows that there will be an optimum dose at which net economic return is maximized.

Calculating an optimum dose. Because fungicide treatment does not instantly reduce pathogen growth and symptom expression, the $x$ and $y$ axes of step 1 are measured at different times. A consequence is that fungicide treatments have to be applied during

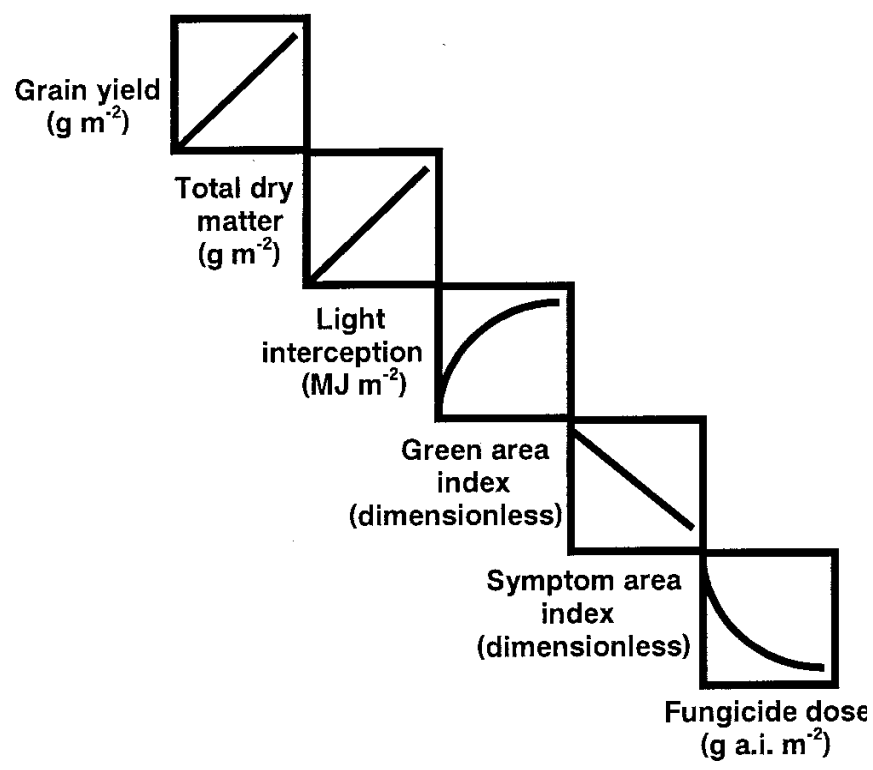

Fig. 1. Diagrammatic representation of the steps linking fungicide input to yield output for a hypothetical crop of winter wheat affected by foliar disease. The dependent variable from one relationship becomes the independent variable for the next. 
canopy expansion (the foundation period) in order to protect green area after anthesis (the yield forming period). To calculate an optimum dose, the benefit of fungicide treatment can be considered as accumulating throughout the yield forming period. It is unlikely that all the quantities represented by the parameters in equation 1 would remain constant during that time. In particular, GAI $\left(L_{h}\right)$ will decline to zero due to natural senescence, and disease $\left(S_{a}\right.$ and $S_{b}$ ) will increase.

Data from an undiseased wheat canopy, presented by Green and Ivins (16), suggest that the decline in green area postanthesis might be considered as approximately constant with time. Royle (38) noted that leaves infected by a pathogen as they emerge do not show symptoms until sporulating lesions develop one latent period after infection, and in many pathosystems, during canopy expansion several new leaves can emerge in the space of one latent period. Hence, disease in the upper canopy is unlikely to reduce green area substantially until canopy expansion ceases, shortly before anthesis. Therefore, healthy and diseased canopies will be considered as sharing a common maximum GAI at anthesis. Clearly, analysis of pathosystems, other than foliar diseases of wheat, may require substantially different simplifying assumptions.

The effect of foliar disease can be represented most simply as an increase in the rate of GAI loss, leading to a shortening of canopy duration. The effect of fungicide treatment is to ameliorate the loss, thus maintaining canopy duration and grain filling. More complex functions to describe green area loss could be incorporated in the analysis, but this would compromise the derivation of an analytical solution. We therefore adhere to this simple description of disease progress and will consider later whether greater sophistication would affect the conclusions materially.

Here, $L_{h}, S_{a}$, and $S_{b}$ are considered state variables, the first declining from a maximum value at anthesis, and the latter two increasing from zero at anthesis; the rates of change of $S_{a}$ and $S_{b}$, and hence the time at which GAI $=0$, are dose dependent.

If $L_{h}, S_{a}$, and $S_{b}$ are taken to change linearly from known values at anthesis, they need only be quantified at one time during the yield forming period. Here, $L_{h \text { max }}$ represents GAI at anthesis, and $\mathrm{YFP}_{h}$ is the yield forming period for a healthy crop, which, by definition, is the time taken for GAI to decrease from $L_{h \text { max }}$ to zero in the absence of disease. There is evidence (R. SylvesterBradley, unpublished data) that the time taken for GAI to decrease to zero, in the absence of disease, is substantially independent of $L_{h \text { max }}$; thus, the end of $\mathrm{YFP}_{h}$ was taken as the reference point for disease variables $S_{a}$ and $S_{b}$.

In the future, it may be possible to increase precision by accounting for changes in $\varepsilon_{p}, I_{p}, k_{1}, k_{2}$, and $D$ through time. The limitations of current literature dictate that these should be considered parameters that remain constant throughout the yield forming period, for each case being considered. Similarly, $\phi_{g}$ is taken here as one, but recognizing that this simplification may be unacceptable for the analysis of other pathosystems (particularly of indeterminate crops), the parameter is retained in the derivation below.

In order to integrate fractional interception $(f)$ over time, GAI at any time $(t)$ can be calculated as $L=L_{h \text { max }}-Z t$, where $Z$ is the rate of GAI loss given by

$$
Z=\left[L_{h \max }-D\left(S_{a}+S_{b} e^{-k_{2} \cdot \operatorname{dose}}\right)\right] / \mathrm{YFP}_{h}
$$

substituting the function for $L$ into Beer's law:

$$
f=1-e^{-k_{1}\left(L_{h \max }-Z t\right)}
$$

and integrating with respect to $t$ gives

$$
F=t-\left(e^{-k_{1} L_{h \max }} / k_{1} Z\right) e^{k_{1} Z t}
$$

The integrated fractional interception $(F)$ from $t=0$ (anthesis) to $t_{\text {gai }=0}$ (the time when senescence is complete) is the expression calculated with $t=t_{\mathrm{gai}=0}$ minus the expression calculated with $t=$ 0 , therefore

$$
F=\left[t_{g a i=0}-\left(e^{-k_{1} L_{h \max }} / k_{1} Z\right) e^{k_{1} Z t_{g a i=0}}\right]-\left[0-\left(e^{-k_{1} L_{h \max }} / k_{1} Z\right)\right]
$$

where $t_{\text {gai }=0}=L_{h \text { max }} / Z$. Substituting in the function for $t_{\text {gai }=0}$ and simplifying gives

$$
F=(1 / Z)\left[L_{h \text { max }}+\left(e^{-k_{1} L_{h \max }} / k_{1}\right)\left(1-e^{k_{1} L_{h \max }}\right)\right]
$$

and

$$
\text { Grain } \mathrm{DM}=\phi_{g} \varepsilon_{p} I_{p} F_{i}
$$

where $I_{p}$ is incident PAR per day.

Substituting in the function for $Z$ and rearranging into three terms to give each a biological meaning

Grain DM $=\left[\phi_{g} \varepsilon_{p} I_{p} \mathrm{YFP}_{h}\right]\left[\frac{L_{h \max }+\frac{e^{-k_{1} L_{\text {max }}}}{k_{1}}\left(1-e^{k_{1} L_{\text {max }}}\right)}{L_{h \max }}\right]\left[\frac{L_{h \max }}{L_{h \text { max }}-D\left(S_{a}+S_{b} e^{-k_{2} \text { dose }}\right)}\right]$

where the first, second, and final terms (in square parentheses), respectively, represent (i) yield if all incident radiation were intercepted, (ii) the proportion of incident radiation intercepted by a healthy canopy, and (iii) the proportion of the healthy interception, intercepted by the green area remaining in a diseased canopy at a given dose.

Equation 7 can be considered in the form of an inverse exponential, with a constant numerator that incorporates Beer's law, and a denominator that incorporates the dose-response function

$$
\text { Grain } \mathrm{DM}=\alpha /\left(\beta+\gamma e^{-\delta \text { dose }}\right)
$$

where

$$
\alpha=\phi_{g} \varepsilon_{p} I_{p} \operatorname{YFP}_{h}\left[L_{h \max }+\left(e^{-k_{1} L_{h \max }} / k_{1}\right)\left(1-e^{k_{1} L_{h \max }}\right)\right]
$$

and $\beta=L_{h \max }-\left(D S_{a}\right), \gamma=-D S_{b}$, and $\delta=k_{2}$. The optimum economic dose is equated with the point on the yield on dose curve at which the cost of an increase in dose is equal to the benefit through increase in yield, i.e., when $d$ grain $\mathrm{DM} / d$ dose $=R$, where $R$ is the ratio of unit of fungicide cost over unit of value of grain dry matter.

Differentiating equation 8 gives

$$
\left(\alpha \gamma \delta e^{-\delta \text { dose }}\right) /\left(\beta+\gamma e^{-\delta \text { dose }}\right)^{2}=R
$$

which can be rearranged to make optimum dose the dependent variable:

$$
\text { Optimum dose }=\frac{\log _{e} \frac{-(2 R \beta-\alpha \delta)-\sqrt{-4 R \beta \alpha \delta+\alpha^{2} \delta^{2}}}{2 R \gamma}}{-\delta}
$$

Sensitivity analysis. Equation 10 provides a means of identifying those parameters likely to be most influential in determining the optimum dose. The temporal and spatial boundaries, simplifying assumptions, and parameter ranges taken for this analysis are broadly compatible with the way Septoria tritici (anamorph of Mycosphaerella graminicola) interacts with the leaf canopy of wheat in the United Kingdom.

The analysis was constrained to a hypothetical crop canopy occupying $1 \mathrm{~m}^{2}$ of ground during the yield forming period; however, the same principles apply whether fungicide dose decisions are being made for a field, or a small part of a field using precision agriculture technology to account for in-field variation. 
It was assumed that there was no translocation to grain of assimilates accumulated during earlier phases of growth, and that sink capacity (9) was not limiting to grain growth.

Data to quantify step 1 were taken from field experiments in which a single fungicide treatment was applied just as the flag leaf emerged. All of the effect of this treatment on disease and GAI was assumed to have been expressed between anthesis and canopy senescence. Therefore, all of the benefit of treatment could be attributed to the yield forming period.

Maximum, minimum, and mean values for each parameter and state variable were taken from the literature to be representative of the grain filling period of wheat in the United Kingdom (Table 1). In the absence of published data, variation in the degree to which symptom area underestimated green area loss $(D)$ was set within the biologically plausible range from 0 to $50 \%$ underestimation.

Disease symptoms cannot be estimated reliably from assessments made at the end of the yield forming period because they become difficult to distinguish from natural senescence. Therefore, estimates for the dose-response parameters were calculated for the end of $\mathrm{YFP}_{h}$ with percentage of disease and total leaf area values from assessments halfway through the yield forming period (33). The percent disease measurements were of Septoria blotch on the upper four leaves, for a range of seven triazole and chlorophenyl single active ingredient fungicides, applied as a single spray at growth stage 37 (GS37) to GS39 (49) to a highly susceptible cultivar at two sites in three seasons. In the absence of associated leaf area index data, these percent values were converted to SAI units using the mean value for total canopy size taken halfway through the yield forming period, assuming that canopy size in the absence of disease would not vary substantially with fungicide dose.

Fungicide manufacturers set the recommended dose and price primarily according to biological activity rather than manufacturing costs per gram of active ingredient. The amount of active ingredient per recommended dose for the seven fungicides considered here, varied from 0.008 to $0.1 \mathrm{~g}$ a.i. $\mathrm{m}^{-2}$. Therefore, $k_{2}$ (the incremental increase in activity per dose unit) and $R$ will tend to interact to a small or great extent if they are expressed in relation to proportional or absolute dose units, respectively. For the sensitivity analysis, $R$ and $k_{2}$ were therefore expressed using proportions of the recommended dose. The ratio of fungicide cost over grain value was calculated with commercial fungicide costs for the range of active ingredients from which the $S_{b}, S_{a}$, and $k_{2}$ values were derived and a typical grain value (expressed per gram of dry matter). Spray application costs were excluded.

Each variable or parameter was changed throughout its range, while keeping all others fixed at their means, and the optimum dose was calculated. Minimum, mean, and maximum values for all parameters and variables are given in Table 1.
Error propagation analysis. Propagation of errors is a concern in models in which a desired $y$ variable (in this case yield, or ultimately, optimum dose) is predicted in several steps in such a way that the model variables used to predict $y$ are themselves predicted variables (17). An exhaustive analysis of uncertainty is beyond the scope of this paper, but error in the prediction of the optimum dose was quantified according to the standard error $\left(\Delta a_{i}\right)$ of each input parameter or state variable $\left(a_{i}\right)$, in the conventional way

$$
(\Delta \text { optimum dose })^{2}=\left(\frac{d o p t}{d a_{1}} \Delta a_{1}\right)^{2}+\left(\frac{d o p t}{d a_{2}} \Delta a_{2}\right)^{2}+\cdots+\left(\frac{d o p t}{d a_{n}} \Delta a_{n}\right)^{2}
$$

This simple method assumes that there are no correlations or, more precisely, covariances between parameters or state variables, and that errors are normally distributed.

\section{RESULTS}

With parameters and state variables set to mean values, disease reduced canopy duration by 11 days (Fig. 2A). A single fungicide treatment was able to retrieve only approximately half of that loss. The dose-response curve state variables $\left(S_{a}\right.$ and $\left.S_{b}\right)$, which were quantified in the GAI dimension, were expressed in the analysis through an exponential change in canopy duration, with each dose increment having a decreasing effect and the rate of change of canopy duration with dose being determined by the rate of change in SAI with dose $\left(k_{2}\right)$. The rate of decline in fractional interception was slow immediately postanthesis and accelerated with constantly diminishing GAI (Fig. 2B), as dictated by Beer's law.

The response curve of yield on dose was of inverse exponential form, rising toward an upper asymptote determined by $\alpha$ and $\beta$ (equation 8). The yield dose-response curve represented the disease dose-response curve in step 1 , inverted by the negative slope $(D)$ of the relationship between SAI and GAI loss in step 2 and scaled according to the relationship between disease and yield represented by the parameters quantifying extinction coefficient $\left(k_{1}\right)$, radiation use efficiency $\left(\varepsilon_{p}\right)$, and dry matter partitioning $\left(\phi_{g}\right)$, in steps 3 to 5 .

Unpreventable disease, represented as the lower asymptote of step 1 by $S_{a}$, translated into the yield dose-response curve as a substantial disparity between the upper yield asymptote $\left(Y_{\mathrm{dose}=\infty}=\right.$ $\left.588 \mathrm{~g} \mathrm{~m}^{-2}\right)$ and yield in the absence of disease $\left(Y_{\text {undiseased }}=690 \mathrm{~g} \mathrm{~m}^{-2}\right.$, with all parameters and state variables at mean values, except $S_{a}$ and $S_{b}$ set to zero, and yields quoted at $85 \%$ dry matter for comparison with field data). The attainable yield (32) was further constrained by the recommended dose, which acts as a statutory maximum dose. But with the mean value for $k_{2}$ representing a level of dose-response curvature commensurate with good fungicide efficacy, $Y_{\text {dose=1 }}$ was only $5 \mathrm{~g} \mathrm{~m}^{-2}$ less than $Y_{\text {dose= }=\infty}$. The pre-

TABLE 1. Model parameters and state variables minimum, means, and maximum for sensitivity analysis

\begin{tabular}{|c|c|c|c|c|c|}
\hline Parameter $^{\mathrm{a}}$ & Minimum $(-1)$ & Means (0) & Maximum (1) & Units & Source \\
\hline$R$ & 14.0 & 19.5 & 25.0 & Dimensionless & This study \\
\hline$\varepsilon_{p}$ & 1.6 & 2.4 & 3.2 & $\mathrm{~g} \mathrm{MJ}^{-1}$ PAR & (12; M. J. Foulkes, unpublished data) \\
\hline$I_{p}$ & 6.5 & 8.5 & 10.5 & MJ PAR m ${ }^{-2}$ day $^{-1}$ & (45; A. Gay, unpublished data) \\
\hline$k_{1}$ & 0.37 & 0.46 & 0.55 & Dimensionless & $(48)$ \\
\hline$D$ & -1.00 & -1.25 & -1.50 & Dimensionless & This study \\
\hline$k_{2}$ & 1.10 & 2.95 & 4.80 & $\mathrm{~m}^{2}$ dose $\mathrm{e}^{-1}$ & (33) \\
\hline $\mathrm{YFP}_{h}$ & 28 & 42 & 56 & Days & (45) \\
\hline \multicolumn{6}{|c|}{ State variable ${ }^{b}$} \\
\hline$L_{h \max }$ & 3.8 & 6.5 & 9.2 & Dimensionless & (A. Gay, unpublished data) \\
\hline$S_{a}$ & 0.3 & 0.9 & 1.5 & Dimensionless & $(33)$ \\
\hline$S_{b}$ & 0.3 & 1.0 & 1.7 & Dimensionless & (33) \\
\hline
\end{tabular}

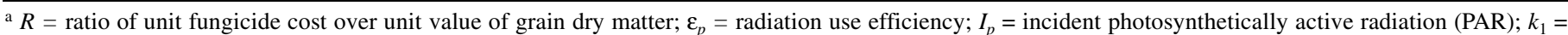
extinction coefficient for PAR; $D=$ ratio of green area index $(\mathrm{GAI})$ loss to symptom area index $(\mathrm{SAI}) ; k_{2}=$ rate of change in $\mathrm{SAI}$ with dose; and $\mathrm{YFP} h=$ yield forming period for healthy crop.

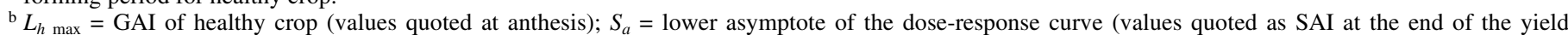
forming period); and $S_{b}=$ the amount of SAI that might potentially be controlled with an infinite dose, therefore $S_{a}+S_{b}=$ untreated SAI . 
ventable yield loss $\left(Y_{\text {dose }=1}-Y_{\text {dose }=0}\right)$ was $77 \mathrm{~g} \mathrm{~m}^{-2}$, and the economically preventable yield loss $\left(Y_{\text {dose }=\text { opt }}-Y_{\text {dose }=0}\right)$ was $74 \mathrm{~g} \mathrm{~m}^{-2}$.

Minimum, mean, and maximum values (Table 1) for all parameters and state variables are represented on the $x$ axis of the sensitivity diagram (Fig. 3) by $-1,0$, and 1 , respectively. The results of the sensitivity analysis for optimum dose, for any particular parameter or state variable, were dependent on both the range selected for that parameter or state variable and the means selected for the others, and may have been affected by correlations. However, the differences in sensitivity were of such magnitude (Fig. 3) that identification of the parameters of importance was unlikely to have been compromised by inappropriate selection of ranges and means. Similarly, consideration of the biology represented by each pairwise combination of parameters or state variables suggests that, with two exceptions considered later, they are unlikely to be correlated substantially.

As dictated by the form of equation 10, the relationships of optimum dose on $R, D, S_{b}, I_{p}, \mathrm{YFP}_{h}$, and $\varepsilon_{p}$ were exponential (the curve for $\mathrm{YFP}_{h}$ coincided with that for $\varepsilon_{p}$ ), with optima declining toward an asymptote in relation to $R$ and rising toward an asymptote in relation to the other parameters as parameter values increased (Fig. 3). Of this group, the amount of SAI that might potentially be controlled by an infinite dose $\left(S_{b}\right)$ was the most influential by a large margin, followed in order of decreasing slope, and hence influence, by $\varepsilon_{p}$ (or $\left.\mathrm{YFP}_{h}\right), R, I_{p}$, and $D$.

The relationships of optimum dose on $S_{a}, L_{h \max }, k_{1}$, and $k_{2}$ were more complex. The dose-response curvature parameter $\left(k_{2}\right)$ was substantially the most influential of this group. Decreasing doseresponse curvature caused increasing dose optima to compensate for poorer disease control per unit of active ingredient. Generally, $S_{a}$ and $L_{h \text { max }}$ were inversely related to the optimum dose. However, there was evidence of a slight reduction in the optimum as the $L_{h \text { max }}$ minimum was approached, because ultimately, the optimum must equal zero if there is no canopy to protect ( $L_{h \text { max }}$ equals zero). $k_{1}$ was the least influential parameter.

The parameters and state variables affected the optimum dose via the yield-response curve. For a given ratio of unit of fungicide cost over unit value of grain dry matter $(R)$, the optimum was determined jointly by the potentially preventable yield loss $\left(Y_{\mathrm{dose}=\infty}-\right.$ $\left.Y_{\text {dose }=0}\right)$ and the curvature of the yield response to dose $\left(\delta=k_{2}\right)$. Small potentially preventable yield losses (representing low untreated disease, high unpreventable disease, or low yield loss per unit of disease) and strong curvature (representing high pathogen sensitivity to the fungicide) led to low optimum doses and vice versa.

Two potential correlations deserve consideration. Firstly, for any given level of untreated disease, $S_{a}$ and $S_{b}$ will be inversely
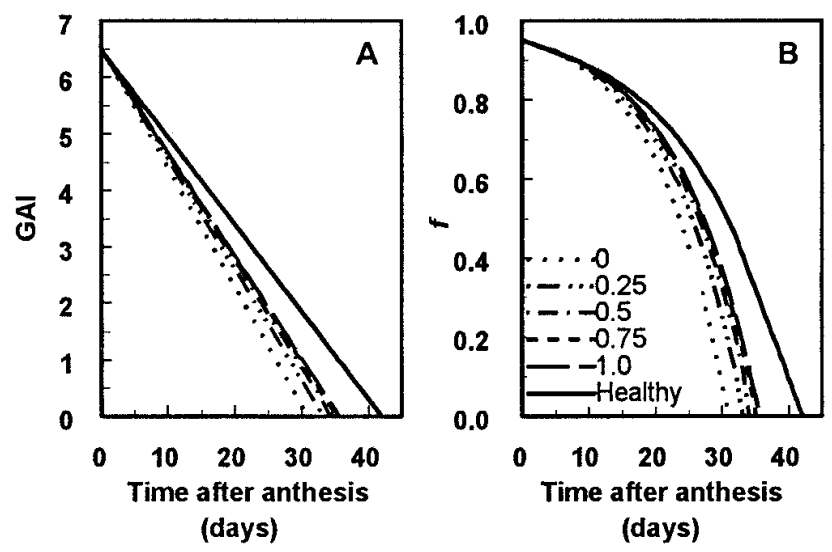

Fig. 2. A, Green area index (GAI) and $\mathbf{B}$, fractional interception decline curves postanthesis for diseased canopies at $0,0.25,0.5,0.75$, and 1.00 proportions of recommended dose and a healthy canopy. All parameters and state variables are set to means. related. These state variables affect the optimum dose in opposite senses, but $S_{b}$ was substantially more influential. Therefore, for a given level of untreated disease $\left(S_{a}+S_{b}\right)$, any change in the proportion that could potentially be prevented by fungicide treatment, $S_{b} /\left(S_{a}+S_{b}\right)$, resulted in a change in the optimum dose that was positively associated with the change in $S_{b}$. Similarly, for any given controllable proportion of the disease, the optimum dose related positively to untreated disease via $S_{b}$. Secondly, for some pathosystems, large canopies (high $L_{h \text { max }}$ ) resulting from high levels of nitrogen uptake are more likely to suffer severe disease $(7,25,26)$, therefore $S_{a}$ and $S_{b}$ may be positively associated with $L_{h \text { max }}$. The influence of $L_{h \max }$ on the optimum was small relative to that of $S_{b}$. Therefore, although $L_{h \text { max }}$ and $S_{b}$ are negatively and positively associated with the optimum, respectively, high nitrogen levels are likely to increase the optimum dose via the effect on the amount of potentially controllable symptom area $\left(S_{b}\right)$.

Comparisons of calculated optimum doses with recommended doses need to be treated with some caution, because the means and ranges for the parameters and state variables relating to fungicide cost and performance (namely $R, k_{2}, S_{a}$, and $S_{b}$ ) describe a population of fungicides rather than a particular combination of values representing a specific product, and parameter and state variables were obtained from disparate sources. Nevertheless, the results of the sensitivity analysis were in good accordance with expectations. The optimum at mean values was a little below the recommended dose at 0.84 . Optima higher than the recommended dose were only indicated where dose-response curvature $\left(k_{2}\right)$ was poor or the amount of potentially controllable symptom area $\left(S_{b}\right)$ was high.

The nature of equation 10 suggests numerous interactions between combinations of parameters and state variables. Consider for example, combinations of the most influential parameter and state variable, $k_{2}$ and $S_{b}$. The efficacious fungicide with high doseresponse curvature represented by the maximum for $k_{2}$ had an optimum dose below the recommended dose even when untreated disease severity was high (represented by $S_{b}$ at its maximum). As $k_{2}$ decreased from the maximum toward the mean, the effect was to increase the optimum dose at higher values of $S_{b}$ but leave the optimum at minimum $S_{b}$ largely unchanged. As $k_{2}$ decreased below the mean, optima with high $S_{b}$ continued to increase, but optima with minimum $S_{b}$ decreased. For a fungicide with little dose-response curvature, represented by the minimum $k_{2}$ value, there was a relatively narrow range of $S_{b}$ values (between the minimum and the mean) that gave optimum doses below the recommended dose. With higher disease severities, doses greater than the legal limit would be required to optimize economic output from the use of fungicides with poor dose efficiency.

Standard error (SE) values for most of the parameters and state variables were available from the literature and unpublished data cited in Table 1 . All SE values were $<10 \%$ of the mean value, with the exception of $k_{2}$ and $S_{b}$, of which larger SE values were associated with dose-response curves for some fungicides. To allow a representative estimate of total error relating to optimum dose and direct comparisons of the contributions of errors in its components, the SE for each parameter and state variable was set at $10 \%$ of the mean value. Dose and $R$ were taken as "known" values without error. The SE of the optimum dose, with all parameters and state variables set at their means, was \pm 0.084 . Rerunning the analysis with the SE for each parameter or state variable, in turn, set to zero demonstrated that the influence of each component on total error in the optimum dose was positively associated with its influence as shown by sensitivity analysis (Fig. 3). Hence, errors associated with $L_{h \text { max }}$ and $S_{a}$ were the least influential (SE of optimum dose reduced by 0.001 when SE for $L_{h \text { max }}$ or $S_{a}$ reduced to zero), whereas $k_{2}$ was most influential (SE reduced by 0.016).

To compare the analytical results with empirical data, optimum doses and SE (Table 2) were derived by fitting exponential dose- 
response functions to yield data from field experiments (35). In each of two sites and two seasons, yields of susceptible cv. Riband were measured after single applications of the triazole fungicide tebuconazole (commercial product 'Folicur') at GS37 to GS39, at doses ranging from nil to full recommended dose in increments of 0.25 . The data were comparable to the sensitivity analysis results, because Riband was the cultivar used in the experiments from which the $S_{a}, S_{b}$, and $k_{2}$ parameter ranges and means were derived. The optimum dose estimated from fitting to mean data across the four experiments was in close agreement with the sensitivity analysis $(0.90 \pm 0.10$ and 0.84 , respectively). In addition, the range of the four optima related well to the range from the sensitivity analysis ( 0.38 to 1.54 and 0.43 to 1.32 , respectively), although two of the measured values were poorly estimated and one lay outside the range of doses tested. There were insufficient ancillary observations to test whether differences in optima within the range were adequately explained by the analysis. Mean observed yields at dose $=0$ and 1 were 792 and $878 \mathrm{~g} \mathrm{~m}^{-2}$, respectively, compared with corresponding analytical values of 506 and $583 \mathrm{~g} \mathrm{~m}^{-2}$. The disparity between the observed and analytical values may be explained by the translocation of assimilate to grain from stem reserves accumulated preanthesis (1). A mean value for translocatable stem reserves in 'Riband' has been reported as $240 \mathrm{~g} \mathrm{~m}^{-2}$ (45).

\section{DISCUSSION}

The intention in deriving the steps was that they should describe the crop-pathogen-fungicide system in a way that balanced the level of complexity adopted for each of the components, and that their scope should be sufficiently broad to include the main factors relevant to dose decisions but sufficiently constrained to be testable (30).

The results imply that the dose required to optimize economic returns varies substantially between sites, seasons, and cultivars because of variation in the relationships of dose to disease and disease to yield. Variation will be determined in the former relationship primarily by facets of disease severity $\left(S_{b}\right)$ and fungicide efficacy $\left(k_{2}\right)$, and in the latter by assimilate partitioning $\left(\phi_{g}\right)$.

Below, we consider current understanding of each of the influential parameters and state variables in turn, with a view to focusing experimentation and observation.

Disease severity. Future symptom area, in the absence of treatment, is one determinant of $S_{b}$, the other is the symptom area which cannot be prevented $\left(S_{a}\right)$. The ranges adopted for the sensitivity analysis resulted from experiments where fungicide treatment was applied at a fixed growth stage and, therefore, variation in $S_{b}$ arose predominantly from variation in untreated disease severity across sites and seasons. However, where there is variation in spray timing, the resulting changes in $S_{a}$ may be as influential in determining $S_{b}$ as variation in untreated severity (34). Whereas factors affecting disease severity have long been a focus of research (for example, in relation to $S$. tritici epidemics [41]), factors affecting the proportion of disease that might be controlled are poorly quantified. Low $S_{a}$ values are obtained by well-timed sprays (34), therefore, counter to intuition, good spray timing results in a higher optimum dose. When fungicide application is delayed beyond the optimum timing, for example by adverse weather conditions for spraying, our analysis suggests that increasing the dose in an attempt to obtain effective control would only exacerbate the economic loss. Optima higher than the recommended dose were indicated in the sensitivity analysis where $S_{b}$ was high. Under high disease conditions resulting in high $S_{b}$, or where other combinations of parameters and state variables result in optima greater than one, a single spray treatment would not be sufficient to optimize economic return.

The impact of $S_{b}$ on green area was scaled by the $D$ parameter. The absence of published data on which to base the range for the sensitivity analysis makes interpretation of the influence of shrinkage and defoliation uncertain. However, when leaves of temperate cereals have senesced, either naturally or due to the effects of disease, they remain largely intact but occupy only a small proportion of their original area, suggesting that shrinkage must be substantial. Some of the problems associated with symptom measurement could be avoided by expressing the dose-response curve with $y$-axis units of GAI and removing step 2. Alternatively, measurements of symptom area and green area through time, would allow both axes to be expressed in absolute or area index units (44).

Fungicide efficacy. Both the disease dose-response and Beer's law steps (steps 1 and 3, respectively) are exponential and act in concert to cause curvature of the yield on dose relationship, on which the position of the optimum dose depends. However, the sensitivity analysis revealed that although dose-response curvature $\left(k_{2}\right)$ was highly influential in determining the optimum, the curvature $\left(k_{1}\right)$ of Beer's law was not. The extent to which the two exponential steps influence the curvature of the response of yield to dose depends on their respective $k$ values and the range of variation in their independent variables. The combination of canopy expansion (38), the asymptotic nature of disease progress curves and finite leaf life, ensures that, even in the absence of fungicide treatment, symptoms can only affect a proportion of the total canopy area. A single spray application can prevent only a proportion of symptom expression on a proportion of the whole crop canopy (34). Hence, varying the independent variable for the dose response across the full range from zero to the recommended dose can only vary GAI, the independent variable of Beer's law, within a limited range. This, combined with the respective magnitudes of $k_{1}$ and $k_{2}$, explains why the majority of the curvature in yield response arises from the response in symptom area rather than Beer's law.

Despite early recognition of the importance of variation in fungicide dose-response curves against foliar pathogens (20), there are few published data describing the extent and causes of

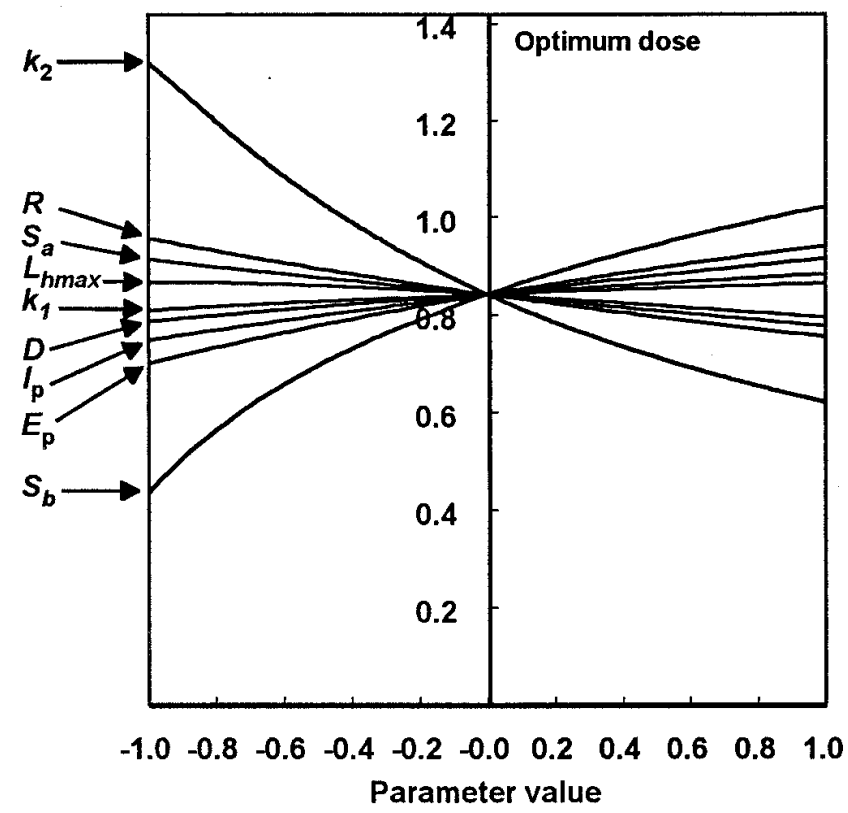

Fig. 3. Sensitivity of optimum fungicide dose (expressed as a proportion of the recommended dose) to each of the parameters and state variables in Equation 10. Table 1 provides the minimum ( -1$)$, mean $(0)$, and maximum (1) values. Parameter definitions: $R=$ ratio of unit fungicide cost over unit value of grain dry matter; $\varepsilon_{p}=$ radiation use efficiency; $I_{p}=$ incident photosynthetically active radiation (PAR); $k_{1}=$ extinction coefficient for PAR; $D=$ ratio of green area index (GAI) loss to symptom area index (SAI); and $k_{2}=$ rate of change in SAI with dose. State variable definitions: $L_{h \max }=$ GAI of healthy crop; $S_{a}=$ lower asymptote of the dose-response curve; and $S_{b}=$ the amount of SAI that might potentially be controlled with an infinite dose, therefore $S_{a}+S_{b}=$ untreated SAI. 
variation, and there are none in absolute units (44). The large number of field plots required to measure comparative doseresponse curves, and the labor intensive nature of absolute measurements, discourage such work. The latter problem has been largely overcome (5), but the difficulty of measuring $k_{2}$ with sufficient precision to provide reliable estimates of optimum dose remains. Testing a wider range of doses than that tested in the field data presented here would improve precision.

The importance of precision was emphasized by the error propagation analysis. Given typical parameter and state variable SE values, total error in the estimation of optimum dose was acceptably smaller than the mean and the range of systematic variation. However, errors in $S_{b}$ and $k_{2}$ were most influential in determining the total error and most prone to error so it is important to seek ways of improving their estimation. The small influence of error in $S_{a}$ on total error suggests that the covariance between $S_{a}$ and $S_{b}$, which was not accounted for in the analysis of error, is unlikely to be important. Hakanson (17) noted that precision is lost at all steps in step-by-step predictive models, so models of this kind should use the fewest possible steps and be subject to critical tests of predictive power before use. Hence, the removal of step 2 , as previously discussed, may have an added advantage of improving precision.

In the sensitivity analysis, optima higher than the recommended dose were indicated where $k_{2}$ was small. Low $k_{2}$ values represented the activity of fungicides with poor activity against $S$. tritici, so adequate control would be either unachievable or would require illegal doses. In practice, more effective fungicides would be selected where $S$. tritici was the predominant target. High efficacy results in lower optima via $k_{2}$.

Fungicide application costs were excluded from the analysis because their only effect is to reduce the net economic output equally at every dose except zero. An effect on decisions would only occur when the net economic benefit from the optimum dose lies between zero and the application cost. In these circumstances, application costs make an otherwise marginally economic application uneconomic.

Assimilate partitioning. Because the analysis was constrained to the yield forming period, all assimilate was assumed to form grain $\left(\phi_{g}=1\right)$. However, it is clear that partitioning must be highly influential, because the optimum dose equals zero if $\phi_{g}$ equals zero.

There are many reports of dry matter in different plant parts, taken sequentially through the season, from healthy wheat crops $(1,12,16,18,39)$. These emphasize the overwhelming shift in recipient organs for assimilate during growth, from roots and leaves, to stems and inflorescences, then grain. Hence, fungicide application is unlikely to be worthwhile until upper leaves, which will contribute substantially to photosynthesis after anthesis, have emerged.

The precise boundary between the foundation and yield forming periods is blurred by the accumulation of assimilates, particularly

TABLE 2. Optimum doses and standard errors derived by fitting exponential dose-response functions to yield data from field experiments ${ }^{\mathrm{a}}$

\begin{tabular}{lccc}
\hline Site & Harvest year & $\begin{array}{c}\text { Optimum } \\
\text { dose }\end{array}$ & $\begin{array}{c}\text { Standard } \\
\text { error }\end{array}$ \\
\hline Morley, Norfolk & 1995 & 1.14 & 0.07 \\
& 1996 & 0.38 & 0.07 \\
Rosemaund, Herefordshire & 1994 & 0.81 & 0.61 \\
& 1996 & 1.54 & 1.24 \\
& Mean $^{\text {b }}$ & 0.90 & 0.10 \\
\hline
\end{tabular}

${ }^{\mathrm{a}}$ In each of two sites and two seasons, yields of susceptible cv. Riband were measured following a single application of the triazole fungicide tebuconazole at growth stage 37 (GS37) to GS39, at doses ranging from nil to full recommended dose in increments of 0.25 .

${ }^{\mathrm{b}}$ Optimum dose and standard error calculated from curves fitted to mean data. in the stem, for later translocation to grain. The weight of soluble stem carbohydrate increases rapidly after GS31 $(4,45)$, as stems extend, and reaches a peak shortly after flowering. However, soluble stem carbohydrate represents a minor proportion of the total above ground DM at anthesis (45), so for healthy crops, $\phi_{g}$ must remain relatively small until shortly before flowering. This leaves open to further study, the possibility of greater contributions from stored assimilates when the host is stressed by disease (15).

One explicit assumption in the sensitivity analysis was that the capacity of the grain sink was not limiting to yield. If this were not the case, the yield response to disease control would be subject to an upper limit determined by sink capacity, resulting in a lower optimum dose. There is no point preserving the capacity to generate carbohydrate for which there is no store. Waggoner and Berger's (52) analysis of a range of contrasting pathosystems suggests that sink limitation is not a major concern in crop species that have been subjected to intensive selection and breeding for high yield. However, it is likely that certain environments and genotypes will conspire to create sink-limited crops. In addition, there is some evidence that early disease can affect sink capacity, for example, by reducing the number of fertile florets (47). Predictive precision may therefore be prejudiced unless assessment of sink capacity in absolute units becomes possible.

Disease and yield. Zadoks (53) reviewed the sometimes contradictory evidence for linkage between attainable yield and diseaseinduced yield loss. Cases were described in which yield losses for a given severity were proportional to, or independent of, attainable yield. These cases are explicable through equation 7, in which the first two terms combine to quantify attainable yield in the absence of the biotic stress of disease, as defined by Nutter et al. (32), and the final term defines the impact of disease. The form of the equation suggests that, in general, crops capable of higher attainable yield in the absence of disease would tend to suffer greater diseaseinduced losses. But the relationship between yield in the absence of disease and disease-induced loss would vary substantially, according to the parameter combinations, which represented particular pathosystems. A sensitivity analysis for yield, with disease variates set to zero, gave the biologically plausible result that grain DM was particularly sensitive to variation in $I_{p}, \varepsilon_{p}$, and $\mathrm{YFP}_{h}$. Paradoxically, the most influential parameters in determining yield in the absence of disease were among the least influential in determining the rate of change of yield with dose and, hence, the dose optimum for diseased crops. An optimistic implication of this degree of independence, between yield potential and the optimum dose, is that future progress with predicting the need for fungicide treatment might not be seriously compromised by the slow progress in improving prediction of yield (24).

Madden and Nutter (27) concluded that, for type I pathosystems, even small changes in RUE would be directly manifested as yield reduction. Our analysis supports the view that disease-induced reductions in RUE would affect yield, but suggests that the change would have to be substantially larger than that measured between contrasting healthy crops to materially affect the optimum dose. Therefore, the observation, from field measurements of gas exchange, that a wide range of pathogens had a damaging influence beyond the lesion area (42) may have relatively little impact on the need for treatment. Rabbinge et al. (36) suggested that loss of efficiency might occur with the first small increments of disease. If the loss occurs at severity values less than $S_{a}$ and does not increase with subsequent increments of severity, then the limitations of fungicide efficacy mean that the loss of efficiency is unpreventable and will not markedly influence the optimum dose. However, for any pathosystem in which the effect on efficiency is both large and changes with severity, $\varepsilon_{p}$ should be made dependent on SAI. Alternatively, "virtual lesions" (2) could be invoked to account for effects of disease that extend beyond the visible symptoms. 
It might have been anticipated that the size of the canopy at anthesis $\left(L_{h \max }\right)$ would have been influential in determining the optimum dose, because a unit of green area loss due to disease would have less influence on fractional interception on large canopies, where the slope defined by Beer's law was shallow, than on small canopies. The optimum dose declined with increasing $L_{h \text { max }}$ but only to a limited extent. The predominant reason for low influence being that disease-induced GAI loss was small early in the yield forming period, when the contrast between small and large canopies was greatest, and large close to canopy senescence, when the contrast was minimal. It is possible that incorporating the delay in the postanthesis onset of healthy senescence, that is sometimes evident in temperate wheat crops, in the form of the analysis, would increase the influence of the canopy parameters.

Our analysis of the joint effects of natural senescence and disease opposes the belief that, for type II pathosystems, crop growth and yield would be little affected until disease-induced reduction in GAI was pronounced (27). This belief arises from Beer's law, which predicts that disease would have to reduce GAI to substantially less than that giving close to full interception, before interception was affected markedly. However, natural senescence ensures that even large canopies will lose sufficient green area toward the end of the yield forming period to render fractional interception highly sensitive to disease-induced green area loss. Therefore, natural senescence increases the impact of a unit of disease on fractional interception, and disease accelerates senescence, bringing light interception and hence yield formation to a premature end.

The spray timings at which greatest benefits accrue per unit dose are determined largely by culm leaf emergence (34), so the task for those seeking to improve fungicide treatment decisions is to predict the facets of disease severity, fungicide efficacy, and assimilate partitioning represented here by $S_{b}, k_{2}$, and $\phi_{g}$. Neglect of any of these will prejudice both the accuracy and precision of dose optimization, with important consequences. The slope of the response curve of yield value minus fungicide cost on fungicide dose will generally be steeper below than above the optimum. Therefore, where there is uncertainty about the optimum, economic prudence demands over treatment. In this study, we propose the term "appropriate dose" to mean the optimum dose for a given set of circumstances. Epidemiologists, crop protectionists, and physiologists should aim to minimize uncertainty so the dose applied can be consistently close to the appropriate dose.

\section{ACKNOWLEDGMENTS}

This work was funded by the U.K. Ministry of Agriculture, Fisheries and Food, and the Home-Grown Cereals Authority. We thank our many colleagues at the University of Nottingham and ADAS Center for Research in Agronomy, specifically A. Gay, J. Foulkes, and C. Dyer for calculating ranges for parameter estimates.

\section{LITERATURE CITED}

1. Austin, R. B., Edrich, J. A., Ford, M. A., and Blackwell, R. D. 1977. The fate of DM, carbohydrates and ${ }^{14} \mathrm{C}$ lost from leaves and stems of wheat during grain filling. Ann. Bot. 45:309-319.

2. Bastiaans, L. 1991. Ratio between virtual and visual lesion size as a measure to describe reduction in leaf photosynthesis of rice due to leaf blast. Phytopathology 81:611-615.

3. Boote, K. J., Jones, J. W., Mishoe, J. W., and Berger, R. D. 1983. Coupling pests to crop growth simulators to predict yield reductions. Phytopathology 73:1581-1587.

4. Borrell, A. K., Incoll, L. D., Simpson, R. J., and Dalling, M. J. 1989. Partitioning of dry matter and the deposition and use of stem reserves in a semi-dwarf wheat crop. Ann. Bot. 63:527-539.

5. Bryson, R. J., Paveley, N. D., Clark, W. S., Sylvester-Bradley, R., and Scott, R. K. 1997. Use of in-field measurements of green leaf area and incident radiation to estimate the effects of yellow rust epidemics on the yield of winter wheat. Eur. J. Agron. 7:53-62.

6. Carver, T. L. W., and Griffiths, E. 1981. Relationship between powdery mildew infection, green leaf area and grain yield of barley. Ann. Appl. Biol. 99:255-266

7. Daniel, D. L., and Parlevliet, J. E. 1995. Effects of nitrogen fertilization on disease severity and infection type of yellow rust on wheat genotypes varying in quantitative resistance. J. Phytopathol. 143:679-681.

8. Dimond, A. E., Heuberger, J. W., and Stoddard, E. M. 1941. Role of the dosage-response curve in the evaluation of fungicides. Conn. Agric. Exp. Stn. Bull. 451:635-667.

9. Evans, L. T., and Wardlaw, I. F. 1996. Wheat. Pages 501-518 in: Photoassimilate Distribution in Plants and Crops. Source-Sink Relationships. E. Zamski and A. A. Schaffer, eds. Marcel Dekker Inc., New York.

10. Ferrandino, F. J. 1989. Spatial and temporal variation of a defoliating plant disease and reduction in yield. Agric. For. Meteorol. 47:273-289.

11. Finney, J. 1993. Risks and rewards from lower chemical inputs into agriculture. Proc. HGCA Cereals R\&D Conf., Home-Grown Cereals Authority, London.

12. Gallagher, J. N., and Biscoe, P. V. 1978. Radiation absorption, growth and yield of cereals. J. Agric. Sci. 91:47-60.

13. Garry, G., Jeuffroy, M. H., Ney, B., and Tivoli, B. 1998. Effects of Ascochyta blight (Mycosphaerella pinodes) on the photosynthesizing leaf area and the photosynthetic efficiency of the green leaf area of dried pea (Pisum sativum). Plant Pathol. 47:473-479.

14. Gaunt, R. E. 1995. The relationship between disease severity and yield. Annu. Rev. Phytopathol. 33:119-144.

15. Gaunt, R. E., and Wright, A. C. 1992. Disease-yield loss relationships in barley. II. Contribution of stored stem reserves to grain filling. Plant Pathol. 41:688-701.

16. Green, C. F., and Ivins, J. D. 1984. Late infestations of take-all (Gaeumannomyces graminis var. tritici) on winter wheat (Triticum aestivum cv. Virtue): Yield, yield components and photosynthetic potential. Field Crops Res. 8:199-206.

17. Hakanson, L. 1999. Error propagation in step-by-step predictions: Examples for environmental management using regression models for lake ecosystems. Environ. Model. Softw. 14:49-58.

18. Hay, R. K. M. 1995. Harvest index: A review of its use in plant breeding and crop physiology. Ann. Appl. Biol. 126:197-216.

19. Hooker, A. L. 1979. Estimating disease losses based on the amount of healthy leaf tissue during the plant reproductive period. Genetica 11: 181-192.

20. Horsfall, J. G. 1945. Fungicides and Their Action. Chronica Botanica, Waltham, MA.

21. Jeger, M. J. 1986. The potential of analytic compared with simulation approaches to modelling in plant disease epidemiology. Pages 255-281 in: Plant Disease Epidemiology: Population Dynamics and Management. K. J. Leonard and W. E. Fry, eds. Macmillan Publishing Company, New York.

22. Johnson, K. B. 1987. Defoliation, disease, and growth: A reply. Phytopathology 77:1495-1497.

23. Johnson, K. B., and Teng, P. S. 1990. Coupling a disease progress model for early leaf blight to a model of potato growth. Phytopathology 80: 416-425.

24. Landau, S., Mitchell, R. A. C., Barnett, V., Colls, J. J., Craigon, J., Moore, K. L., and Payne, R. W. 1998. Testing winter wheat simulation models' predictions against observed UK grain yields. Agric. For. Meteorol. 89:85-99.

25. Last, F. T. 1953. Some effects of temperature and nitrogen supply on wheat powdery mildew. Ann. Appl. Biol. 40:312-322.

26. Leitch, M. H., and Jenkins, P. D. 1995. Influence of nitrogen on the development of Septoria epidemics in winter wheat. J. Agric. Sci. (Cambridge) 124:361-368.

27. Madden, L. V., and Nutter, F. W. 1995. Modelling crop losses at the field scale. Can. J. Plant Pathol. 17:124-137.

28. Madeira, A. C., and Clark, J. A. 1994. Disease and resource capture. Pages 167-188 in: Resource Capture by Crops. J. L. Monteith, R. K. Scott, and M. H. Unsworth, eds. Nottingham University Press, Nottingham.

29. Monteith, J. L. 1977. Climate and the efficiency of crop production in Britain. Philos. Trans. R. Soc. Lond. Ser. B 281:277-294.

30. Monteith, J. L. 1996. The quest for balance in crop modelling. Agron. J. 88:695-697.

31. Monteith, J. L., and Unsworth, M. H. 1990. Principles of Environmental Physics. Edward Arnold, London.

32. Nutter, F. W., Jr., Teng, P. S., and Royer, M. H. 1993. Terms and concepts for yield, crop loss and disease thresholds. Plant Dis. 77:211-215.

33. Paveley, N. D., and Hims, M. J. 1998. Appropriate fungicide doses for winter wheat, experiment 1: Dose responses for conazole and morpholine fungicides. Project report 166. Home-Grown Cereals Authority, London.

34. Paveley, N. D., Lockley, K. D., Vaughan, T. B., Thomas, J., and Schmidt, K. 2000. Predicting effective fungicide doses, through observation of 
leaf emergence. Plant Pathol. 49:748-766.

35. Paveley, N. D., and Stevens, D. B. 1998. Appropriate fungicide doses for winter wheat, experiment 2: Dose-response by variety interactions. Project report 166. Home-Grown Cereals Authority, London.

36. Rabbinge, R., Jorritsma, I. T. M., and Schans, J. 1985. Damage components of powdery mildew in winter wheat. Neth. J. Plant Pathol. 91: 235-247.

37. Rouse, D. I. 1988. Use of crop growth-models to predict the effects of disease. Annu. Rev. Phytopathol. 26:183-201.

38. Royle, D. J. 1994. Understanding and predicting epidemics. Plant Pathol. 43:777-789.

39. Schnyder, H. 1993. The role of carbohydrate storage and redistribution in the source-sink relations of wheat and barley during grain filling. New Phytol. 123:233-246.

40. Secher, B. J. M., and Jorgensen, L. N. 1995. Current developments in fungicide use - success or failure? Pages 241-250 in: A Vital Role for Fungicides in Cereal Production. H. G. Hewitt, D. Tyson, D. W. Hollomon, J. M. Smith, W. P. Davies, and K. R. Dixon, eds. Bios Scientific Publishers, Oxford.

41. Shaw, M. W., and Royle, D. J. 1993. Factors affecting the severity of epidemics of Mycosphaerella graminicola (Septoria tritici) on winter wheat in the UK. Plant Pathol. 42:882-899.

42. Shtienberg, D. 1992. Effects of foliar diseases on gas exchange processes: A comparative study. Phytopathology 82:760-765.

43. Spitters, C. J. T., Roermund, H. J. W., Nassau, H. G. M. G., van, Schepers, J., and Mesdag, J. 1990. Genetic variation in partial resistance to leaf rust in winter wheat: Disease progress, foliage senescence and yield reduction. Neth. J. Plant Pathol. 96:3-15.
44. Sylvester-Bradley, R., Paveley, N. D., Gladders, P., and Scott, R. K. 1998. Towards an absolute basis for assessment and prediction of foliar disease. Page 3.1.5S in: Proc. Int. Congr. Plant Pathol., 7th., Vol. 1.

45. Sylvester-Bradley, R., Scott, R. K., Clare, R. W., Kettlewell, P. S., Kirby, E. J. M., Stokes, D. T., Weightman, R. M., Gillett, A. G., Macbeth, J. E., Gay, A., Foulkes, M. J., Spink, J. H., Hoad, S. H., Russell, G., Mills, A., Duffield, S. J., and Crout, N. M. J. 1997. The Wheat Growth Guide. Home-Grown Cereals Authority, London.

46. Thomas, M. R., Garthwaite, D. G., and Banham, A. R. 1996. Pesticide usage survey report 141: Arable farm crops in Great Britain 1996. Minist. Agric. Fisher. Food, London.

47. Thomson, W. J., and Gaunt, R. E. 1986. The effect of speckled leaf blotch on apical development and yield in winter wheat in New Zealand. Ann. Bot. 58:39-48.

48. Thorne, G. N., Pearman, I., Day, W., and Todd, A. D. 1988. Estimation of radiation interception by winter wheat from measurements of leaf area. J. Agric. Sci. 110:101-108.

49. Tottman, D. R. 1987. The decimal code for the growth stages of cereals, with illustrations. Ann. Appl. Biol. 110:441-454.

50. van Oijen, M. 1990. Photosynthesis is not impaired in healthy tissue of blighted potato plants. Neth. J. Plant Pathol. 96:55-63.

51. Van Roermund, H. J. W., and Spitters, C. J. T. 1990. Simulation of yield reduction by leaf rust in winter wheat, applied to the analysis of genetic variation in partial resistance. Neth. J. Plant Pathol. 96:17-28.

52. Waggoner, P. E., and Berger, R. D. 1987. Defoliation, disease, and growth. Phytopathology 77:393-398.

53. Zadoks, J. C. 1985. On the conceptual basis of crop loss assessment: The threshold theory. Annu. Rev. Phytopathol. 23:455-473. 